\title{
A random field model for generating synthetic microstructures of functionally graded materials
}

\author{
Sharif Rahman*, ${ }^{*} \ddagger$ \\ College of Engineering, The University of Iowa, Iowa City, IA 52242, U.S.A.
}

\begin{abstract}
SUMMARY
This article presents a new level-cut, inhomogeneous, filtered Poisson random field model for representing two-phase microstructures of statistically inhomogeneous, functionally graded materials with fully penetrable embedded particles. The model involves an inhomogeneous, filtered Poisson random field comprising a sum of deterministic kernel functions that are scaled by random variables and a cut of the filtered Poisson field above a specified level. The resulting level-cut field depends on the Poisson intensity, level, kernel functions, random scaling variables, and random rotation matrices. A reconstruction algorithm including model calibration and Monte Carlo simulation is presented for generating samples of two-phase microstructures of statistically inhomogeneous media. Numerical examples demonstrate that the model developed is capable of producing a wide variety of two- and three-dimensional microstructures of functionally graded composites containing particles of various sizes, shapes, densities, gradations, and orientations. An example involving finite element analyses of random microstructures, leading to statistics of effective properties of functionally graded composites, illustrates the usefulness of the proposed model. Copyright (C) 2008 John Wiley \& Sons, Ltd.
\end{abstract}

Received 17 May 2007; Revised 21 January 2008; Accepted 6 February 2008

KEY WORDS: filtered Poisson field; level-cut field; statistically inhomogeneous media; reconstruction algorithm, Monte Carlo simulation

\section{INTRODUCTION}

Mathematical models involving Voronoi tessellations [1], regular lattices [2], random checkerboards [3], random fields [4-8], and others [2] are frequently used to simulate random microstructures of heterogeneous materials and calculate their effective material properties. Many

\footnotetext{
*Correspondence to: Sharif Rahman, Department of Mechanical and Industrial Engineering, The University of Iowa, Iowa City, IA 52242, U.S.A.

†E-mail: rahman@engineering.uiowa.edu

$\ddagger$ Professor.

Contract/grant sponsor: U.S. National Science Foundation; contract/grant number: CMS-0409463
}

Copyright (C) 2008 John Wiley \& Sons, Ltd. 
reconstruction algorithms entailing Monte Carlo simulation have been developed to generate samples of two-phase random media [2]. An essential step in all reconstruction algorithms is the selection of a microstructure model based on statistics obtained from available measurements. For example, the selection of probabilistic models for two-phase, particle-matrix composites is usually based on estimates of the phase volume fraction and the two-point correlation function. Information on particle geometry can also be incorporated in the model selection process, for instance, a lineal-path function $[9,10]$. Random field models derived from non-linear transformations with the memory of Gaussian fields have also been proposed to capture higher-order statistics and describe a broader range of two-phase microstructures [11].

Level-cut random fields, defined by cuts of random fields above the specified levels or outside the bounded intervals, are currently employed for simulating random microstructures [2]. It is typically assumed that the random field that is cut is a homogeneous Gaussian field [5,6]. The resulting field, known as the level-cut Gaussian random field, however, is non-Gaussian whether or not the initial field is Gaussian. Available measurements of microstructure properties provide information on some features of the level-cut Gaussian field models, which may be limited to estimates of their mean and correlation functions. The selection of a level-cut Gaussian field for a microstructure with specified second-moment properties poses at least two problems. First, it is not possible to uniquely determine a level-cut field from its mean and correlation functions, i.e. second-moment properties alone, as the field is non-Gaussian. Such a lack of uniqueness in modeling microstructures under partial information has been illustrated by Yeong and Torquato [9, 10]. Second, there may not be a Gaussian field such that its level-cut image contains the target second-moment properties. This is because the correlation structure of the Gaussian field may not even exist if the second-moment properties of the level-cut field are specified arbitrarily [12]. Alternative level-cut fields, defined as cuts of a homogeneous, filtered Poisson field, have been proposed to circumvent some of these problems, leading to a wide variety of two-phase microstructures [8]. More recently, non-Gaussian random field models entailing a hard-core Poisson field and spherical harmonics have also appeared [13]. However, most existing models are meant for statistically homogeneous media, where the mean and correlation functions of the random field are spatially and translationally invariant, respectively. In statistically inhomogeneous media, such as those encountered in functionally graded materials (FGMs) [14-17], porous media [18], and others [19], the probabilistic characteristics of the random field depend on the absolute values of its argument. Therefore, a practical need clearly exists for modeling statistically inhomogeneous microstructures. Unfortunately, research in microstructure modeling of inhomogeneous media has not been widespread and is only now gaining attention. For example, a noteworthy work by Quintanilla and Torquato presents microstructure functions for modeling statistically inhomogeneous two-phase media comprising fully penetrable spherical particles in a matrix [20]. While such works are encouraging and constitute a step in the right direction, more general probabilistic models of microstructures are required, where embedded particles in a matrix can have random size, orientation, and/or shape characteristics.

This article presents a level-cut, inhomogeneous, filtered Poisson random field model for representing two-phase microstructures of FGMs. The model is based on (1) an inhomogeneous, filtered Poisson random field comprising a sum of deterministic kernel functions that are scaled by random variables and centered at Poisson points and (2) a cut of the Poisson field above a specified level. Section 2 describes some fundamental properties of a generic inhomogeneous random field. Section 3 presents the proposed random field, including a calibration procedure for matching target statistics from the measurements of actual microstructures. A Monte Carlo algorithm is proposed 
to generate samples of the level-cut, inhomogeneous, filtered Poisson field. Numerical examples illustrating the proposed model and its usefulness in stochastic evaluations of effective properties are presented in Section 4. The limitations of the model and future work are discussed in Section 5. Finally, the conclusions are given in Section 6.

\section{RANDOM FIELD}

Let $(\Omega, \mathscr{F}, P)$ be a probability space, where $\Omega$ is the sample space, $\mathscr{F}$ is the $\sigma$-algebra of subsets of $\Omega$, and $P$ is the probability measure. Defined on the probability space and indexed by a spatial coordinate $\mathbf{x} \in \mathscr{D} \subset \mathbb{R}^{d}$, where $d \geqslant 1$ is an integer and $\mathscr{D}$ is a bounded subset of $\mathbb{R}^{d}$, consider a real-valued inhomogeneous random field $Z(\mathbf{x}): \mathscr{D} \times \Omega \rightarrow \mathbb{R}$. The probability law of $Z$ is completely defined by its $n$ th-order finite-dimensional distribution, i.e. the joint distribution of the random vector $\left\{Z\left(\mathbf{x}_{1}\right), \ldots, Z\left(\mathbf{x}_{n}\right)\right\}$, where $n \geqslant 1$ is an arbitrary integer, $\mathbf{x}_{i} \in \mathscr{D}$, and $i=1, \ldots, n$. The $n$ th-order distribution is the probability of $\bigcap_{i=1}^{n}\left\{Z\left(\mathbf{x}_{i}\right) \leqslant z_{i}\right\}$, where $z_{i} \in \mathbb{R}$. Unlike a homogeneous random field, the $n$ th-order distribution of $Z$ depends on the absolute values of arguments $\mathbf{x}_{1}, \ldots, \mathbf{x}_{n}$.

Denote by $\mathscr{L}_{2}(\Omega, \mathscr{F}, P)$, or simply $\mathscr{L}_{2}$, a collection of random variables $Z$ for each $\mathbf{x} \in \mathscr{D}$ such that $\mathbb{E}\left[|Z|^{2}\right]<\infty$, where $\mathbb{E}$ represents the expectation operator. If $Z$ is in $\mathscr{L}_{2}$, then its secondmoment properties, i.e. mean $\mu(\mathbf{x}):=\mathbb{E}[Z(\mathbf{x})]$, correlation function $r(\mathbf{x}, \mathbf{u}):=\mathbb{E}[Z(\mathbf{x}) Z(\mathbf{u})]$, and covariance function $c(\mathbf{x}, \mathbf{u}):=\mathbb{E}[(Z(\mathbf{x})-\mu(\mathbf{x}))(Z(\mathbf{u})-\mu(\mathbf{u}))]$, exist and are finite. Again, unlike a homogeneous random field, the second-moment properties of $Z$ also depend on the absolute values of arguments $\mathbf{x}$ and $\mathbf{u}$.

\section{PROPOSED MODEL}

\subsection{Inhomogeneous, filtered Poisson field}

Filtered Poisson processes or fields are characterized by pulses of random or deterministic shapes and random magnitudes arriving at Poisson points in a bounded Borel set of $\mathbb{R}^{d}$. These processes or fields have been successfully applied to various science and engineering disciplines, including mechanics, physics, astronomy, earthquake engineering, finance, and others [12].

Consider an inhomogeneous, Poisson random field $\mathscr{N}\left(\mathscr{D}^{\prime}\right)$ with an intensity measure $\mu_{\mathscr{D}^{\prime}}:=$ $\int_{\mathscr{D}^{\prime}} \lambda(\mathbf{x}) \mathrm{d} \mathbf{x}$, where $\lambda(\mathbf{x}) \geqslant 0$ is a spatially variant intensity function and $\mathscr{D}^{\prime} \in \mathscr{B}\left(\mathbb{R}^{d}\right)$ is a bounded Borel set. The Poisson point field has the following properties: (1) the number $\mathscr{N}\left(\mathscr{D}^{\prime}\right)$ of points

in a bounded subset $\mathscr{D}^{\prime}$ has the Poisson distribution with intensity measure $\mu_{\mathscr{D}^{\prime}}$ and (2) random variables $\mathscr{N}\left(\mathscr{D}_{1}^{\prime}\right), \ldots, \mathscr{N}\left(\mathscr{D}_{K}^{\prime}\right)$ for any integer $K \geqslant 2$ and disjoint sets $\mathscr{D}_{1}^{\prime}, \ldots, \mathscr{D}_{K}^{\prime}$ are statistically independent. The Poisson field $\mathscr{N}\left(\mathscr{D}^{\prime}\right)$ gives the number of points in $\mathscr{D}^{\prime}$ and is characterized by the probability

$$
P\left[\mathscr{N}\left(\mathscr{D}^{\prime}\right)=n\right]=\frac{\left(\int_{\mathscr{D}^{\prime}} \lambda(\mathbf{x}) \mathrm{d} \mathbf{x}\right)^{n}}{n !} \exp \left(-\int_{\mathscr{D}^{\prime}} \lambda(\mathbf{x}) \mathrm{d} \mathbf{x}\right), \quad n=0,1,2, \ldots
$$

that $n$ Poisson points exist in $\mathscr{D}^{\prime}$. The mean $\mathbb{E}\left[\mathscr{N}\left(\mathscr{D}^{\prime}\right)\right]$ and the variance $\operatorname{var}\left[\mathscr{N}\left(\mathscr{D}^{\prime}\right)\right]$ of $\mathscr{N}\left(\mathscr{D}^{\prime}\right)$ are both equal to $\mu_{\mathscr{D}^{\prime}}:=\int_{\mathscr{D}^{\prime}} \lambda(\mathbf{x}) \mathrm{d} \mathbf{x}$. 
Consider an inhomogeneous, filtered Poisson random field

$$
Z(\mathbf{x}):=\left\{\begin{array}{ll}
0, & \mathscr{N}\left(\mathscr{D}^{\prime}\right)=0 \\
\sum_{i=1}^{\mathscr{N}\left(\mathscr{D}^{\prime}\right)} Z_{i} h\left(\mathbf{R}_{i}\left(\mathbf{x}-\boldsymbol{\Gamma}_{i}\right)\right), & \mathscr{N}\left(\mathscr{D}^{\prime}\right)>0
\end{array}, \quad \mathbf{x} \in \mathscr{D} \subset \mathscr{D}^{\prime} \subset \mathbb{R}^{d}\right.
$$

where $\left\{\boldsymbol{\Gamma}_{i}\right\}$ is a collection of Poisson points, $h: \mathbb{R}^{d} \rightarrow \mathbb{R}$ is a non-negative kernel function, $\left\{Z_{i}\right\}$ is a collection of independent and identically distributed real-valued random variables, and $\left\{\mathbf{R}_{i}\right\}$ is a collection of independent and identically distributed rotation matrices in $\mathbb{R}^{d}$. In Equation (2), both $\mathscr{D}$ and $\mathscr{D}^{\prime}$ are bounded subsets of $\mathbb{R}^{d}$ such that points of $\mathscr{N}$ falling in the set difference $\mathbb{R}^{d} \backslash \mathscr{D}^{\prime}$ do not contribute to the value of $Z$ in $\mathscr{D}$. Also, the subset $\mathscr{D}^{\prime}$ is bounded if, for example, $h$ has a compact support. The filtered Poisson field $Z(\mathbf{x})$ can be viewed as the response of a filter with a transfer function at point $\mathbf{x}$ that is subjected to a collection of random pulses arriving at the Poisson points $\left\{\boldsymbol{\Gamma}_{i}\right\}$.

3.1.1. Characteristic functions and moments. Suppose that the random variable $Z_{i} \in \mathscr{L}_{2}$ and $h$ are square integrable in $\mathbb{R}^{d}$. From Equation (1), $P\left[\mathscr{N}\left(\mathscr{D}^{\prime}\right)=n\right]=\left(\mu_{\mathscr{D}^{\prime}}^{n} / n !\right) \exp \left(-\mu_{\mathscr{D}^{\prime}}\right)$, where $\mu_{\mathscr{D}^{\prime}}:=$ $\int_{D^{\prime}} \lambda(\mathbf{x}) \mathrm{d} \mathbf{x}$. Given $\mathscr{N}\left(\mathscr{D}^{\prime}\right)=n$, the unordered set of points $\boldsymbol{\Gamma}_{1}, \ldots, \boldsymbol{\Gamma}_{n}$ has the same distribution as $n$ independent and identical copies of $\mathbf{V}$ that follows the probability density $\lambda(\mathbf{v}) / \mu_{\mathscr{D}}$ for $\mathbf{v} \in \mu_{\mathscr{D ^ { \prime }}}$ and zero otherwise. By conditioning on $\mathscr{N}\left(\mathscr{D}^{\prime}\right)$, the characteristic function of $Z(\mathbf{x})$ is

$$
\begin{aligned}
& \varphi_{Z(\mathbf{x})}(t):=\mathbb{E}[\exp \{j t Z(\mathbf{x})\}] \\
& =\mathbb{E}\left[\exp \left\{j t \sum_{i=1}^{\mathcal{N}\left(\mathscr{D}^{\prime}\right)} Z_{i} h\left(\mathbf{R}_{i}\left(\mathbf{x}-\boldsymbol{\Gamma}_{i}\right)\right)\right\}\right] \\
& =\sum_{n=0}^{\infty} \mathbb{E}\left[\exp \left\{j t \sum_{i=1}^{\mathscr{N}\left(\mathscr{D}^{\prime}\right)} Z_{i} h\left(\mathbf{R}_{i}\left(\mathbf{x}-\boldsymbol{\Gamma}_{i}\right)\right) \mid \mathscr{N}\left(\mathscr{D}^{\prime}\right)=n\right\}\right] P\left(\mathscr{N}\left(\mathscr{D}^{\prime}\right)=n\right) \\
& =\sum_{n=0}^{\infty} \mathbb{E}\left[\exp \left\{j t \sum_{i=1}^{n} Z_{i} h\left(\mathbf{R}_{i}\left(\mathbf{x}-\boldsymbol{\Gamma}_{i}\right)\right)\right\}\right] P\left(\mathscr{N}\left(\mathscr{D}^{\prime}\right)=n\right) \\
& =\sum_{n=0}^{\infty}\left(\mathbb{E}\left[\exp \left\{j t Z_{1} h\left(\mathbf{R}_{1}(\mathbf{x}-\mathbf{V})\right)\right\}\right]\right)^{n} P\left(\mathscr{N}\left(\mathscr{D}^{\prime}\right)=n\right) \\
& =\sum_{n=0}^{\infty} \frac{\mu_{\mathscr{D}^{\prime}}^{n}}{n !} \exp \left(-\mu_{\mathscr{D}^{\prime}}\right)\left(\int_{\mathscr{D}^{\prime}} \frac{\lambda(\mathbf{v})}{\mu_{\mathscr{D}^{\prime}}} \mathbb{E}\left[\exp \left\{j t Z_{1} h\left(\mathbf{R}_{1}(\mathbf{x}-\mathbf{v})\right)\right\}\right] \mathrm{d} \mathbf{v}\right)^{n} \\
& =\sum_{n=0}^{\infty} \exp \left(-\mu_{\mathscr{D}^{\prime}}\right) \frac{\left(\int_{\mathscr{D}^{\prime}} \lambda(\mathbf{v}) \mathbb{E}\left[\exp \left\{j t Z_{1} h\left(\mathbf{R}_{1}(\mathbf{x}-\mathbf{v})\right)\right\}\right] \mathrm{d} \mathbf{v}\right)^{n}}{n !} \\
& =\exp \left\{\int_{\mathscr{D}^{\prime}} \lambda(\mathbf{v}) \mathbb{E}\left[\exp \left\{j t Z_{1} h\left(\mathbf{R}_{1}(\mathbf{x}-\mathbf{v})\right)\right\}-1\right] \mathrm{d} \mathbf{v}\right\}
\end{aligned}
$$


where $j=\sqrt{-1}$. Taking first- and second-order derivatives of $\varphi_{Z(\mathbf{x})}(t)$ with respect to $t$ yields

$$
\frac{\mathrm{d} \varphi_{Z(\mathbf{x})}(t)}{\mathrm{d} t}=\varphi_{Z(\mathbf{x})}(t) \int_{\mathscr{D}^{\prime}} \lambda(\mathbf{v}) \mathbb{E}\left[\exp \left\{j t Z_{1} h\left(\mathbf{R}_{1}(\mathbf{x}-\mathbf{v})\right)\right\} j Z_{1} h\left(\mathbf{R}_{1}(\mathbf{x}-\mathbf{v})\right)\right] \mathrm{d} \mathbf{v}
$$

and

$$
\begin{aligned}
\frac{\mathrm{d}^{2} \varphi_{Z(\mathbf{x})}(t)}{\mathrm{d} t^{2}}= & \varphi_{Z(\mathbf{x})}(t) \int_{\mathscr{D}^{\prime}} \lambda(\mathbf{v}) \mathbb{E}\left[\exp \left\{j t Z_{1} h\left(\mathbf{R}_{1}(\mathbf{x}-\mathbf{v})\right)\right\} j^{2} Z_{1}^{2} h^{2}\left(\mathbf{R}_{1}(\mathbf{x}-\mathbf{v})\right)\right] \mathrm{d} \mathbf{v} \\
& +\frac{\mathrm{d} \varphi_{Z(\mathbf{x})}(t)}{\mathrm{d} t} \int_{\mathscr{D}^{\prime}} \lambda(\mathbf{v}) \mathbb{E}\left[\exp \left\{j t Z_{1} h\left(\mathbf{R}_{1}(\mathbf{x}-\mathbf{v})\right)\right\} j Z_{1} h\left(\mathbf{R}_{1}(\mathbf{x}-\mathbf{v})\right)\right] \mathrm{d} \mathbf{v}
\end{aligned}
$$

Therefore, the mean $\mu(\mathbf{x})$ and the variance $\sigma^{2}(\mathbf{x})$ of $Z(\mathbf{x})$, derived from the characteristic function and its derivatives in Equations (3)-(5), are

$$
\mu(\mathbf{x}):=\mathbb{E}[Z(\mathbf{x})]=\frac{1}{j} \frac{\mathrm{d} \varphi_{Z(\mathbf{x})}(0)}{\mathrm{d} t}=\mathbb{E}\left[Z_{1}\right] \int_{D^{\prime}} \lambda(\mathbf{v}) \mathbb{E}\left[h\left(\mathbf{R}_{1}(\mathbf{x}-\mathbf{v})\right)\right] \mathrm{d} \mathbf{v}
$$

and

$$
\begin{aligned}
\sigma^{2}(\mathbf{x}):=\mathbb{E}\left[Z^{2}(\mathbf{x})\right]-(\mathbb{E}[Z(\mathbf{x})])^{2} & =\frac{1}{j^{2}} \frac{\mathrm{d}^{2} \varphi_{Z(\mathbf{x})}(0)}{\mathrm{d} t^{2}}-\left(\frac{1}{j} \frac{\mathrm{d} \varphi_{Z(\mathbf{x})}(0)}{\mathrm{dt}}\right)^{2} \\
& =\mathbb{E}\left[Z_{1}^{2}\right] \int_{D^{\prime}} \lambda(\mathbf{v}) \mathbb{E}\left[h^{2}\left(\mathbf{R}_{1}(\mathbf{x}-\mathbf{v})\right)\right] \mathrm{d} \mathbf{v}
\end{aligned}
$$

respectively, where the expectation operator inside the integrals acts on the random rotation matrix $\mathbf{R}_{1}$. Higher-order moments of $Z(\mathbf{x})$ can be obtained in a similar manner, for instance,

$$
\mu_{m}(\mathbf{x}):=\mathbb{E}\left[Z^{m}(\mathbf{x})\right]=\frac{1}{j^{m}} \frac{\mathrm{d}^{m} \varphi_{Z(\mathbf{x})}(0)}{\mathrm{d} t^{m}}
$$

thereby furnishing a moment of an arbitrary order $m \geqslant 1$. These moments can also be derived by evaluating the $m$ th-order cumulant

$$
\chi_{m}(\mathbf{x})=\frac{1}{j^{m}} \frac{\mathrm{d}^{m}}{\mathrm{~d} t^{m}} \ln \varphi_{Z(\mathbf{x})}(0)=\mathbb{E}\left[Z_{1}^{m}\right] \int_{\mathscr{D}^{\prime}} \lambda(\mathbf{v}) \mathbb{E}\left[h^{m}\left(\mathbf{R}_{1}(\mathbf{x}-\mathbf{v})\right)\right] \mathrm{d} \mathbf{v}
$$

of $Z(\mathbf{x})$ first, then recognizing the generic moment-cumulant relationship

$$
1+\sum_{i=1}^{\infty} \frac{(j t)^{i}}{i !} \mu_{i}(\mathbf{x})=\exp \left\{\sum_{i=1}^{\infty} \frac{(j t)^{i}}{i !} \chi_{i}(\mathbf{x})\right\}
$$

and finally expanding in series the exponential function of Equation (10) and equating coefficients of the same powers of $j t$. Following similar arguments, the mixed moments of $\left\{Z\left(\mathbf{x}_{1}\right), \ldots, Z\left(\mathbf{x}_{n}\right)\right\}$, $n \geqslant 1$, can also be formulated, although the algebra associated with their derivations is less simple.

The joint characteristic function of $\left\{Z\left(\mathbf{x}_{1}\right), \ldots, Z\left(\mathbf{x}_{n}\right)\right\}, n \geqslant 1$, is defined as

$$
\varphi_{Z\left(\mathbf{x}_{1}\right) \cdots Z\left(\mathbf{x}_{n}\right)}(\mathbf{t}):=\mathbb{E}\left[\exp \left\{j\left(t_{1} Z\left(\mathbf{x}_{1}\right)+\cdots+t_{n} Z\left(\mathbf{x}_{n}\right)\right)\right\}\right]
$$


which can be further expanded by similar arguments employed in deriving Equation (3). The Fourier transformation of the joint characteristic function yields the joint probability density function of $\left\{Z\left(\mathbf{x}_{1}\right), \ldots, Z\left(\mathbf{x}_{n}\right)\right\}$. The characteristic functions and moments in Equations (3)-(11) provide useful information on the filtered Poisson field $Z(\mathbf{x})$ in Equation (2). If the Poisson counting field $\mathscr{N}\left(\mathscr{D}^{\prime}\right)$ is homogeneous with a constant intensity, the resulting expressions of the characteristic functions and moments simplify significantly, rendering $Z(\mathbf{x})$ to be a weakly homogeneous random field [8]. Unfortunately, these simplified statistical properties do not apply when modeling statistically inhomogeneous media, which is the focus of this paper.

3.1.2. Asymptotic property. A valuable insight can be gained by examining the asymptotic property of the Poisson field. To do so, let $\lambda(\mathbf{x})=c \lambda_{0}(\mathbf{x})$, where $c$ is a constant intensity parameter and denote the second-moment properties $\mu(\mathbf{x})=c \mu_{0}(\mathbf{x})$ and $\sigma^{2}(\mathbf{x})=c \sigma_{0}^{2}(\mathbf{x})$, where Equations (6) and (7) lead to

$$
\mu_{0}(\mathbf{x})=\mathbb{E}\left[Z_{1}\right] \int_{D^{\prime}} \lambda_{0}(\mathbf{v}) \mathbb{E}\left[h\left(\mathbf{R}_{1}(\mathbf{x}-\mathbf{v})\right)\right] \mathrm{d} \mathbf{v}
$$

and

$$
\sigma_{0}^{2}(\mathbf{x})=\mathbb{E}\left[Z_{1}^{2}\right] \int_{D^{\prime}} \lambda_{0}(\mathbf{v}) \mathbb{E}\left[h^{2}\left(\mathbf{R}_{1}(\mathbf{x}-\mathbf{v})\right)\right] \mathrm{d} \mathbf{v}
$$

Consider the standardized random field $\bar{Z}(\mathbf{x}):=(Z(\mathbf{x})-\mu(\mathbf{x})) / \sigma(\mathbf{x})$, which has zero mean and unit variance. The characteristic function of $\bar{Z}(\mathbf{x})$ is

$$
\begin{aligned}
\varphi_{\bar{Z}(\mathbf{x})}(t) & :=\mathbb{E}[\exp \{j t \bar{Z}(\mathbf{x})\}] \\
& =\exp \left\{-j t \frac{\mu(\mathbf{x})}{\sigma(\mathbf{x})}\right\} \mathbb{E}\left[\exp \left\{j t \frac{Z(\mathbf{x})}{\sigma(\mathbf{x})}\right\}\right] \\
& =\exp \left\{-j t c^{1 / 2} \frac{\mu_{0}(\mathbf{x})}{\sigma_{0}(\mathbf{x})}\right\} \varphi_{Z(\mathbf{x})}(t / \sigma(\mathbf{x})) \\
& =\exp \left\{-j t c^{1 / 2} \frac{\mu_{0}(\mathbf{x})}{\sigma_{0}(\mathbf{x})}+\int_{\mathscr{D}^{\prime}} \lambda(\mathbf{v}) \mathbb{E}\left[\exp \left\{\frac{j t Z_{1} h\left(\mathbf{R}_{1}(\mathbf{x}-\mathbf{v})\right)}{\sigma(\mathbf{x})}\right\}-1\right] \mathrm{d} \mathbf{v}\right\} \\
& =\exp \left\{-j t c^{1 / 2} \frac{\mu_{0}(\mathbf{x})}{\sigma_{0}(\mathbf{x})}+\int_{\mathscr{D}^{\prime}} \lambda_{0}(\mathbf{v}) \mathbb{E}\left[\sum_{k=1}^{\infty} \frac{c^{1-k / 2}}{k !}\left\{\frac{j t Z_{1} h\left(\mathbf{R}_{1}(\mathbf{x}-\mathbf{v})\right)}{\sigma_{0}(\mathbf{x})}\right\}^{k}\right] \mathrm{d} \mathbf{v}\right\} \\
& =\exp \left\{-\frac{t^{2}}{2}+O\left(c^{-1 / 2}\right)\right\}
\end{aligned}
$$

which approaches $\exp \left(-t^{2} / 2\right)$, the characteristic function of a standard Gaussian variable, when $c \rightarrow \infty$. Similar derivations reveal that the random vector $\left\{\bar{Z}\left(\mathbf{x}_{1}\right), \ldots, \bar{Z}\left(\mathbf{x}_{n}\right)\right\}$, for any integer $n \geqslant 1$ and arguments $\left(\mathbf{x}_{1}, \ldots, \mathbf{x}_{n}\right)$, also becomes Gaussian asymptotically as $c \rightarrow \infty$. Therefore, the inhomogeneous, filtered Poisson field $Z(\mathbf{x})$ in Equation (2) can be approximated by an inhomogeneous Gaussian random field when $c$ is very large. In other words, the commonly used Gaussian random field can be viewed as a special case of the filtered Poisson random field. 


\subsection{Level-cut, inhomogeneous, filtered Poisson field}

For an increasing function $g: \mathbb{R} \rightarrow \mathbb{R}$, consider a real-valued translation random field

$$
Y(\mathbf{x}):=g(Z(\mathbf{x}))
$$

which describes a memoryless, measurable, non-linear function of a real-valued random field $Z(\mathbf{x})$. The translation field $Y(\mathbf{x})$ is completely determined by the mapping $g$ and $Z(\mathbf{x})$ with statistical properties derived from Equations (3)-(11). Consider a special form of

$$
g(z)=I(z ; a):=\left\{\begin{array}{ll}
1, & z>a \\
0, & z \leqslant a
\end{array}, \quad z \in \mathbb{R}\right.
$$

where $a$ is a deterministic constant, known as level, and $I(z ; a)$ denotes an indicator function. The translation field $Y$ in Equation (15) with $g$ in Equation (16) is referred to as a level-cut random field. Most existing random field models of microstructures are based on level-cut Gaussian fields, where the random field that is cut is a homogeneous Gaussian field. A notable exception is the work of Grigoriu, who proposed a level-cut filtered Poisson field that leads to a broad range of microstructures, although it is statistically homogeneous [8]. The present work, motivated by spatially variant microstructures of FGMs, requires clipping an inhomogeneous random field, which is defined here as an inhomogeneous, filtered Poisson field $Z(\mathbf{x})$, as in Equation (2). The resulting translation field

$$
Y(\mathbf{x}):=I(Z(\mathbf{x}) ; a)
$$

then becomes a level-cut, inhomogeneous, filtered Poisson field, which provides a convenient model for two-phase functionally graded composites. For example, the subsets $\{\mathbf{x} \in \mathscr{D}: Y(\mathbf{x})=1\}$ and $\{\mathbf{x} \in \mathscr{D}: Y(\mathbf{x})=0\}$ define particles (phase 1) and matrix (phase 2), respectively, that can be derived from a $d$-1-dimensional contour of $Z(\mathbf{x})$ at level $a$. Figure $1^{\S}$ depicts a schematic illustration of the two subsets when $d=2$, obtained from a cut (Figure 1(b)) of a generic random field $Z(\mathbf{x})$ (Figure 1(a)). The dark (charcoal) phase indicates particles embedded in the light (light blue) phase representing the matrix. Equation (17) can be generalized by defining an appropriate indicator function with multiple levels. Furthermore, level-cut fields modeled by multiple versions of Equation (17) can be developed to represent multiphase FGMs, including porous composites. In this paper, however, the theoretical formulation and the numerical examples are limited to single level-cut fields and two-phase FGMs.

The second-moment characteristics of the level-cut field $Y(\mathbf{x})$ in Equation (17) are

$$
\mathbb{E}[Y(\mathbf{x})]=P[Z(\mathbf{x})>a]=p_{1}(\mathbf{x})
$$

and

$$
\mathbb{E}\left[Y\left(\mathbf{x}_{1}\right) Y\left(\mathbf{x}_{2}\right)\right]=P\left[Z\left(\mathbf{x}_{1}\right)>a \cap Z\left(\mathbf{x}_{2}\right)>a\right]=p_{11}\left(\mathbf{x}_{1}, \mathbf{x}_{2}\right)
$$

where $p_{1}(\mathbf{x})$ represents the spatially variant volume fraction of phase 1 and $p_{11}\left(\mathbf{x}_{1}, \mathbf{x}_{2}\right)$ denotes the translationally variant two-point correlation function. Higher-order moments of $Y(\mathbf{x})$ defined by

$$
\mathbb{E}\left[Y\left(\mathbf{x}_{1}\right) \cdots Y\left(\mathbf{x}_{k}\right)\right]=P\left[Z\left(\mathbf{x}_{1}\right)>a \cap \cdots \cap Z\left(\mathbf{x}_{k}\right)>a\right]=p_{1 \cdots 1}\left(\mathbf{x}_{1}, \ldots, \mathbf{x}_{k}\right)
$$

\footnotetext{
${ }^{\S}$ For interpretation of color in Figure 1, the reader is referred to the web version of this article. 

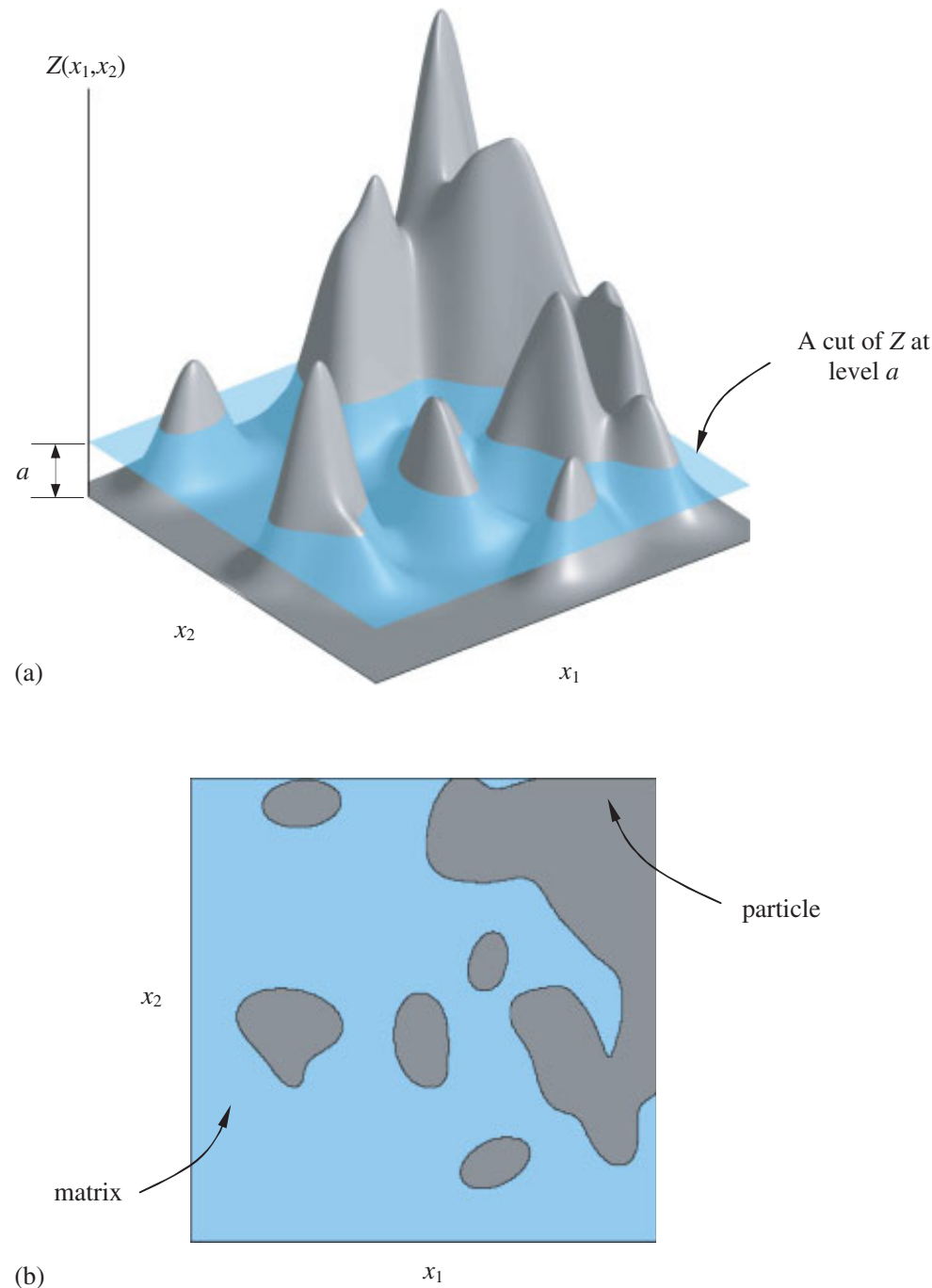

Figure 1. Schematic illustration of a generic level-cut random field in $\mathbb{R}^{2}$ : (a) a sample of $Z\left(x_{1}, x_{2}\right)$ with a cut at level $a$ and (b) two subsets of $Y\left(x_{1}, x_{2}\right)$ obtained from the contour of the sample of $Z\left(x_{1}, x_{2}\right)$ at level $a$.

can also be related to the $k$-point correlation function $p_{1 \cdots 1}\left(\mathbf{x}_{1}, \ldots, \mathbf{x}_{k}\right)$, where $k \geqslant 1$ is a positive integer. As $Y(\mathbf{x})$ is an inhomogeneous field, the correlation function $p_{1 \cdots 1}\left(\mathbf{x}_{1}, \ldots, \mathbf{x}_{k}\right)$ is also spatially variant, i.e. it explicitly depends on the arguments $\mathbf{x}_{1}, \ldots, \mathbf{x}_{k}$. It is not possible to derive analytically the second- or higher-order moments of $Y(\mathbf{x})$ in a general setting, i.e. when $h,\left\{Z_{i}\right\}$, and $\left\{\mathbf{R}_{i}\right\}$ are arbitrarily prescribed. However, these moments are directly related to finite-dimensional distribution functions of $Z(\mathbf{x})$, the statistical properties of which are presented in Section 3.1. 
Let the kernel $h(\xi)$ introduced in Equation (2) be a compactly supported, non-negative function, for example,

$$
h(\xi)=\exp \left(-\xi^{\mathrm{T}} \boldsymbol{\gamma} \boldsymbol{\xi}\right)
$$

where $\xi \in \mathbb{R}^{d}, \sigma_{k}>0$ are some constants, and $\gamma=\operatorname{diag}\left[1 / \sigma_{1}, \ldots, 1 / \sigma_{d}\right] \in \mathbb{R}^{d \times d}$ is a diagonal matrix. Suppose that $\lambda(\mathbf{x}), Z_{i}$, and $h$ are such that the values of $Z$ in a small vicinity $\mathscr{D}\left(\boldsymbol{\Gamma}_{i}\right)$ of $\boldsymbol{\Gamma}_{i}$ can be approximated by

$$
\tilde{Z}(\mathbf{x})=Z_{i} h\left(\mathbf{R}_{i}\left(\mathbf{x}-\Gamma_{i}\right)\right), \quad \mathbf{x} \in \mathscr{D}\left(\boldsymbol{\Gamma}_{i}\right)
$$

leading to an approximate version

$$
\tilde{Y}(\mathbf{x})=I(\tilde{Z}(\mathbf{x}) ; a)=I\left(Z_{i} h\left(\mathbf{R}_{i}\left(\mathbf{x}-\boldsymbol{\Gamma}_{i}\right)\right) ; a\right), \quad \mathbf{x} \in \mathscr{D}\left(\boldsymbol{\Gamma}_{i}\right)
$$

of the level-cut field $Y(\mathbf{x})$ in Equation (17). Therefore, the particles in the microstructure of a two-phase FGMs can be conveniently approximated by the subsets

$$
\left(\mathbf{x}-\boldsymbol{\Gamma}_{i}\right)^{\mathrm{T}} \mathbf{R}_{i}^{\mathrm{T}} \gamma\left(\mathbf{x}-\boldsymbol{\Gamma}_{i}\right) \leqslant-\ln \left(a / Z_{i}\right)
$$

of $\mathscr{D}$ for $Z_{i}>a$ and $\mathbf{x} \in \mathbb{R}^{d}$. Note that the approximate level-cut field $\tilde{Y}(\mathbf{x})$ is applicable when $\lambda(\mathbf{x})$ and $\sigma_{k}$ are both small. While such smallness restriction limits the usefulness of $\tilde{Y}(\mathbf{x})$ somewhat, Equations (23) and (24) can be conveniently employed for finite element discretization of fully penetrable particles even when $\lambda(\mathbf{x})$ and $\sigma_{k}$ are not small. Further explanation is given in the Example section.

\subsection{Reconstruction algorithm}

3.3.1. Model calibration. Consider a two-phase particle-matrix FGM microstructure, which can be modeled by $Y$ in Equation (17), where $Z$ is defined in Equation (2). Suppose that the estimates of some features of $Y$ are available-for instance, the functions $p_{1}(\mathbf{x})$ and $p_{11}\left(\mathbf{x}_{1}, \mathbf{x}_{2}\right)$ and information on the number of particles and particle geometry. The objective of calibration is to determine parameters of the proposed model such that the second moments of $Y$ in Equations (18) and (19), which are also the first- and second-order probability distributions of $Z$, match known target statistics $\bar{p}_{1}(\mathbf{x})$ and $\bar{p}_{11}\left(\mathbf{x}_{1}, \mathbf{x}_{2}\right)$. If estimates of higher-order moments, such as $k$-point correlation functions $\bar{p}_{1 \cdots 1}\left(\mathbf{x}_{1}, \ldots, \mathbf{x}_{k}\right), k \geqslant 3$, are available, they can also be incorporated into the calibration process.

Let $\boldsymbol{\theta}$ define a vector of parameters comprising $\lambda(\mathbf{x}), h, a, Z_{i}$, and $\mathbf{R}_{i}$, which are embedded in the proposed random field model. Using any admissible values of these parameters, Monte Carlo simulation can be employed to generate samples of the random field $Y$ and hence calculate its desired moments. Let $p_{1 \ldots 1}(\cdot ; \boldsymbol{\theta})$ represent the $k$-point correlation function of $Y$ corresponding to a selected value of $\boldsymbol{\theta}$. Denote by $\boldsymbol{\theta}^{*}$ the value of $\boldsymbol{\theta}$ that minimizes the difference between $p_{1 \ldots 1}(\cdot ; \boldsymbol{\theta})$ and the target $\bar{p}_{1 \ldots 1}\left(\mathbf{x}_{1}, \ldots, \mathbf{x}_{k}\right)$ for all $k=1, \ldots, K$ in the least squares or other sense, where $K$ is a user-selected positive integer. The optimization process must consider only feasible values of $\boldsymbol{\theta}$ for which $p_{1 \cdots 1}(\cdot ; \boldsymbol{\theta})$ constitutes a legitimate correlation function. The subspace of feasible values of $\boldsymbol{\theta}$ also depends on the indicator function defined in Equation (16). Most existing models entail calibration processes that are based on matching only the first moment $(K=1)$, i.e. $\bar{p}_{1}(\mathbf{x})$, and sometimes also on matching the second moment $(K=2)$, i.e. $\bar{p}_{11}\left(\mathbf{x}_{1}, \mathbf{x}_{2}\right)$. The proposed model permits a calibration process that can match up to $K$-point correlation functions for any $K \geqslant 1$ with their corresponding estimates, if available. 
Microstructural reconstruction of random heterogeneous materials using specified yet limited correlation functions constitutes an intriguing inverse problem. As $Y(\mathbf{x})$ is non-Gaussian, the statistics $p_{1 \cdots 1}\left(\mathbf{x}_{1}, \ldots, \mathbf{x}_{k}\right), k=1, \ldots, K$, i.e. its 1 - to $K$-point correlation functions, only partially describe its probability law. Consequently, there may exist a collection of random fields, referred to as the class of competing models, that have the same aforementioned statistics. The number of competing models can be reduced if $K$ is theoretically made a large number; however, from a practical viewpoint, the value of $K$ is usually decided based on the available information. Nonetheless, a finite value of $K$, regardless how large, will yield a class of competing models with two or more members. If these members have different sample properties, they will generate microstructures with distinct morphological features, pointing to a lack of uniqueness in any reconstruction algorithm.

3.3.2. Monte Carlo simulation. Recall the inhomogeneous, filtered Poisson field $Z(\mathbf{x})$ defined in Equation (2). The parameters that describe $Z(\mathbf{x})$ are the bounded subsets $\mathscr{D}$ and $\mathscr{D}^{\prime}$ of $\mathbb{R}^{d}$, the probability distribution of $Z_{i}$, the inhomogeneous Poisson field $\mathscr{N}\left(\mathscr{D}^{\prime}\right)$ with spatially variant intensity function $\lambda(\mathbf{x})$, the compactly supported, non-negative function $h$, and the rotation matrix $\mathbf{R}_{i}$. In $\mathbb{R}^{2}$, the rotation matrix for the $i$ th Poisson point in $\mathscr{D}^{\prime}$ is represented by

$$
\mathbf{R}_{i}=\left[\begin{array}{cc}
\cos \Theta_{i} & \sin \Theta_{i} \\
-\sin \Theta_{i} & \cos \Theta_{i}
\end{array}\right], \quad i=1, \ldots, \mathscr{N}, \quad \mathbf{x} \in \mathbb{R}^{2}
$$

where $\Theta_{i}$ 's are independent and identical random variables (rotations) and are uniformly distributed in $[0,2 \pi]$, for example, when particles in a graded material have random orientations with equal probability. The rotation matrix in $\mathbb{R}^{3}$ takes the form

$$
\begin{aligned}
\mathbf{R}_{i}= & {\left[\begin{array}{ccc}
\cos \Psi_{i} & \sin \Psi_{i} & 0 \\
-\sin \Psi_{i} & \cos \Psi_{i} & 0 \\
0 & 0 & 1
\end{array}\right]\left[\begin{array}{ccc}
1 & 0 & 0 \\
0 & \cos \Theta_{i} & \sin \Theta_{i} \\
0 & -\sin \Theta_{i} & \cos \Theta_{i}
\end{array}\right]\left[\begin{array}{ccc}
\cos \Phi_{i} & \sin \Phi_{i} & 0 \\
-\sin \Phi_{i} & \cos \Phi_{i} & 0 \\
0 & 0 & 1
\end{array}\right] } \\
& i=1, \ldots, \mathscr{N}, \quad \mathbf{x} \in \mathbb{R}^{3}
\end{aligned}
$$

where $\Phi_{i}$ 's, $\Theta_{i}$ 's, and $\Psi_{i}$ 's are independent and identical random variables (Euler angles) and are uniformly distributed in $[0,2 \pi],[0, \pi]$, and $[0,2 \pi]$, respectively. If the particle orientations can be controlled or have desired orientations, more appropriate probability distributions of rotations or Euler angles can be prescribed for defining $\mathbf{R}_{i}$.

Once the parameters of the level-cut field $Y(\mathbf{x})$ have been optimized from the calibration process described in the previous subsection, samples of synthetic microstructures of two-phase FGMs can be generated based on the following algorithm:

- Step 1: Define bounded subsets $\mathscr{D}$ and $\mathscr{D}^{\prime}$ of $\mathbb{R}^{d}$. The bounded subset $\mathscr{D}^{\prime}$ must be such that points of $\mathscr{N}$ falling in $\mathbb{R}^{d} \backslash \mathscr{D}^{\prime}$ do not contribute to the value of $Z(\mathbf{x})$ in $\mathscr{D}$. Specify the kernel function $h$-for example, Equation (21) —and define its parameters.

- Step 2: Generate a sample $n^{*}$ of the homogeneous Poisson random variable $N^{*}\left(\mathscr{D}^{\prime}\right)$, which has a constant intensity $\lambda^{*}=\max _{\mathbf{x} \in \mathbb{R}^{d}} \lambda(\mathbf{x})$, where $\lambda(\mathbf{x})$ is a bounded intensity function in $\mathscr{D}^{\prime}$.

- Step 3: Generate $n^{*}$ independent uniformly distributed points in $\mathscr{D}^{\prime}$. Denote these points by $\mathbf{x}_{i}, i=1, \ldots, n^{*}$. 
- Step 4: Perform thinning of the point set obtained in Step 3. In doing so, each point $\mathbf{x}_{i}$, independent of the other, is kept with probability $\lambda\left(\mathbf{x}_{i}\right) / \lambda^{*}$, which is equivalent to discarding the point with probability $1-\lambda\left(\mathbf{x}_{i}\right) / \lambda^{*}$. The resulting point pattern with the size $n^{* *} \leqslant n^{*}$ follows the inhomogeneous Poisson field $\mathscr{N}\left(\mathscr{D}^{\prime}\right)$ with intensity function $\lambda(\mathbf{x})$. Denote these points by $\boldsymbol{\Gamma}_{i}, i=1, \ldots, n^{* *}$.

- Step 5: Generate $n^{* *}$ independent samples of random rotation matrices $\left\{\mathbf{R}_{i}\right\}$ and random variables $\left\{Z_{i}\right\}$.

- Step 6: Calculate the corresponding samples of the random fields $Z(\mathbf{x})$ from Equation (2) and $Y(\mathbf{x})$ from Equation (17) for a specified level $a$. The sample of $Y(\mathbf{x})$ yields a two-phase, statistically inhomogeneous microstructure.

Independent samples of $Z(\mathbf{x})$ and $Y(\mathbf{x})$ are delivered by repeated application of the above-stated algorithm. The resulting samples can be analyzed to estimate various statistics of these random fields.

\section{NUMERICAL EXAMPLES}

\subsection{Example 1}

Consider a two-dimensional $(d=2)$, functionally graded composite with the bounded domain $\mathscr{D}:=$ $(0,10) \times(0,10) \in \mathbb{R}^{2}$, where fully penetrable elliptical or circular particles with random lengths and widths are embedded in the matrix. The aspect ratio, defining the length-to-width ratio of each particle, is deterministic and identical for all particles. For the inhomogeneous Poisson field $\mathscr{N}\left(\mathscr{D}^{\prime}\right)$, three types of intensity functions, defined by

$$
\lambda(\mathbf{x})= \begin{cases}c x_{1} x_{2} & \text { diagonal } \\ c\left[\left(x_{1}-5.25\right)^{2}+\left(x_{2}-5.25\right)^{2}\right] & \text { centrifugal } \\ c \exp \left[-\sqrt{\left(x_{1}-5.25\right)^{2}+\left(x_{2}-5.25\right)^{2}}\right] & \text { anticentrifugal }\end{cases}
$$

represent diagonal, centrifugal, and anticentrifugal variations of the particle locations, where $\mathscr{D}^{\prime}=$ $(0,10.5) \times(0,10.5), \mathbf{x}=\left\{x_{1}, x_{2}\right\}^{\mathrm{T}} \in \mathscr{D}^{\prime} \subset \mathbb{R}^{2}$, and $c$ is the intensity parameter. The kernel function is given by

$$
h(\xi)=\exp \left(-\frac{\xi_{1}^{2}}{\sigma_{1}^{2}}-\frac{\xi_{2}^{2}}{\sigma_{2}^{2}}\right)
$$

where $\xi=\left\{\xi_{1}, \xi_{2}\right\}^{\mathrm{T}} \in \mathbb{R}^{2}, \sigma_{1}=0.2, \sigma_{2}=0.1$ for the diagonally varying intensity function, and $\sigma_{1}=$ $\sigma_{2}=0.1$ for the remaining two intensity functions with centrifugal or anticentrifugal variations. In other words, the particles have elliptical shapes for the diagonal variation or circular shapes for the centrifugal or anticentrifugal variations. For all three intensity functions, the random variable $Z_{i}=10\left|U_{i}\right|$, where $U_{i}$ is a standard Gaussian random variable with zero mean and unit variance. The rotation matrix $\mathbf{R}_{i}$ is defined by Equation (25), where the rotation $\Theta_{i}$ is uniformly distributed in $[0,2 \pi]$.

Figure 2 shows a sample of the filtered Poisson field $Z(\mathbf{x})$ in Equation (2) for a diagonally varying intensity function with the parameter $c=0.1$ entailing low intensity. The number, location, and shapes of the peaks of $Z$ depend on the intensity function, kernel function $h$ defined by Equation (28), and statistical properties of random variables $\left\{\mathbf{R}_{i}\right\}$ and $\left\{Z_{i}\right\}$. Figure 3(a) shows three 


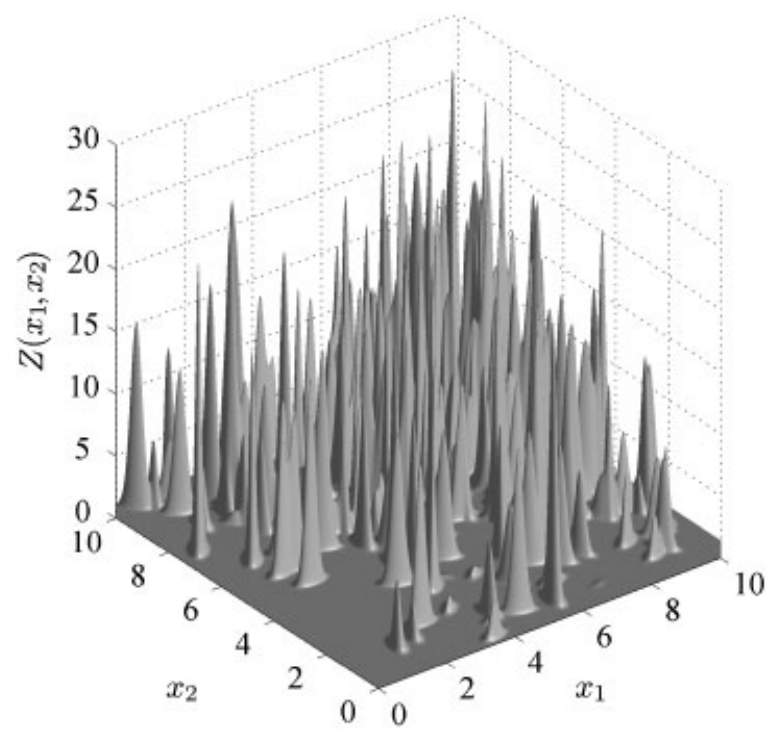

Figure 2. A sample of an inhomogeneous, filtered Poisson field $Z\left(x_{1}, x_{2}\right)$ with a diagonally varying intensity function $\lambda(\mathbf{x})=c x_{1} x_{2} ; c=0.1$.

samples of the level-cut filtered Poisson field $Y(\mathbf{x})$ in Equation (17) obtained from a single sample of $Z(\mathbf{x})$, but three distinct levels, $a=0.1,1$, and 5. They represent synthetic samples of FGM microstructures, where the particles have a target aspect ratio of two, but their sizes are random. Both the number and the sizes of the particles in these composites decrease when the magnitude of level $a$ increases. Therefore, it is possible to calibrate $a$, leading to a synthetic microstructure with the desired statistical properties. If the intensity is sufficiently low, the resulting filtered Poisson field $Z$ can also be approximated by $\tilde{Z}$ in Equation (22). In that case, the geometry of the particles is easy to control as the value of $Z$ at a point $\mathbf{x} \in \mathscr{D}$ depends only on the function $Z_{i} h\left(\mathbf{R}_{i}\left(\mathbf{x}-\Gamma_{i}\right)\right)$ with the center $\Gamma_{i}$ in the vicinity of $\mathbf{x}$. Modeling random microstructures by $\tilde{Y}$, which is a cut field of $\tilde{Z}$, can be viewed as placing particles of random or deterministic shapes at Poisson points in $\mathscr{D}^{\prime}$.

Consider now a slight modification of the graded composites, which still follow a diagonally varying intensity function, but with a higher intensity parameter, $c=0.5$, and three distinct levels, $a=1,5$, and 15. Figure 3(b) shows three samples of $Y(\mathbf{x})$ in Equation (17), obtained again from a single sample of $Z(\mathbf{x})$. Individual particles intersect frequently, particularly for low magnitudes of level $a$. The resulting composites correspond to level-cut filtered Poisson fields of high intensity. If the intensity is sufficiently high, the geometry of the particles will be relatively difficult to control. This is because the value of $Z$ at a point $\mathbf{x} \in \mathscr{D}$ is derived from a sum of functions $Z_{i} h\left(\mathbf{R}_{i}\left(\mathbf{x}-\boldsymbol{\Gamma}_{i}\right)\right)$ with centers $\boldsymbol{\Gamma}_{i}$ in the vicinity of $\mathbf{x}$, so that the excursions of $Z$ above level $a$ depend on all these functions. Nevertheless, the Monte Carlo simulation algorithm described in a previous section can be employed to generate microstructures defined by random fields $Y$ associated with a collection of parameters $\lambda(\mathbf{x}), h, a, Z_{i}$, and $\mathbf{R}_{i}$, and to select values of these parameters that are optimal in some sense.

Finally, Figures 4(a) and (b) demonstrate the versatility of the proposed random field model by depicting FGM microstructures derived from the centrifugal and anticentrifugal intensity functions, respectively. The values of intensity parameter $c$ are 1 for the centrifugal variation and 300 for the 

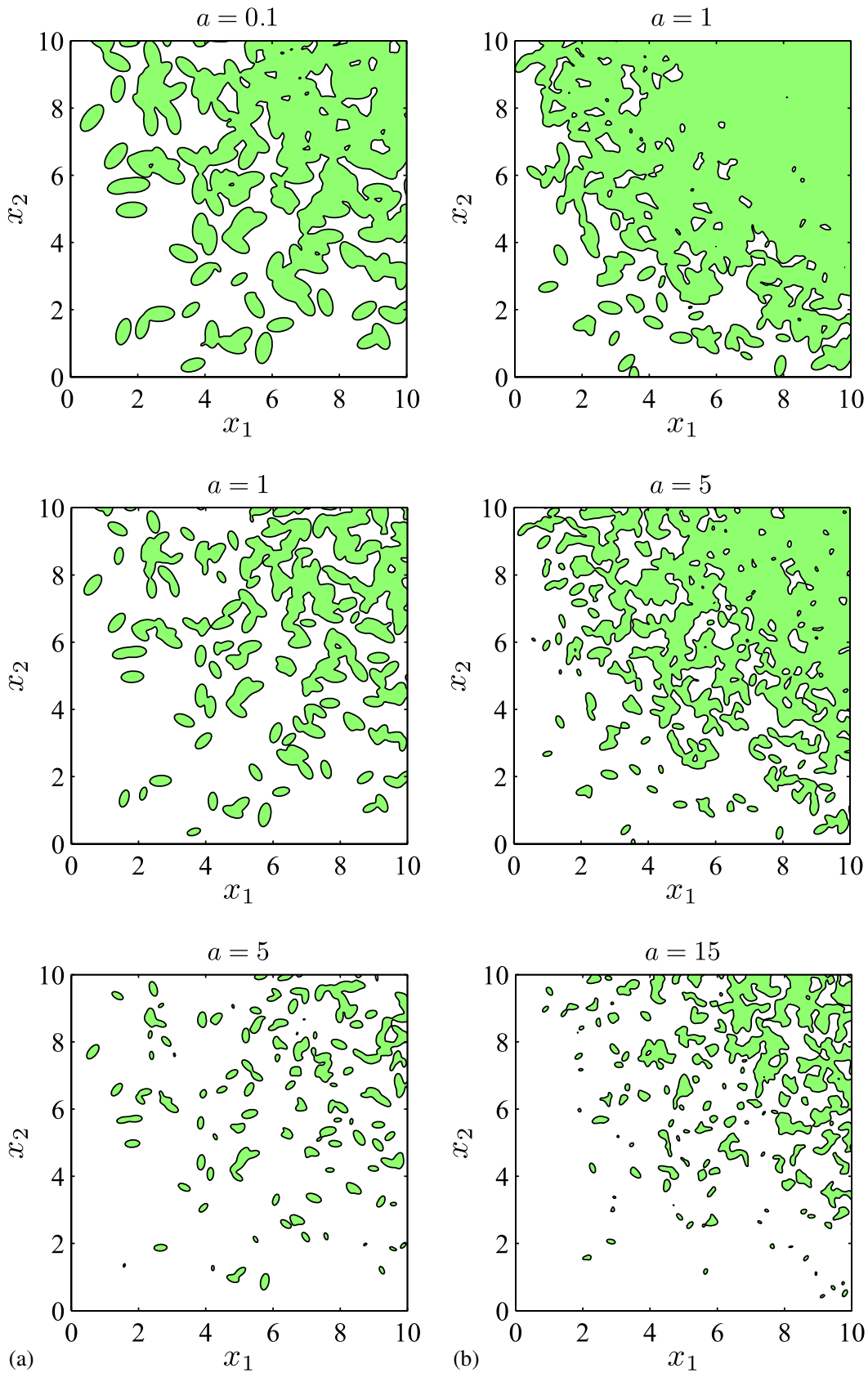

Figure 3. Samples of two-dimensional microstructures of functionally graded composites with a diagonally varying intensity function $\lambda(\mathbf{x})=c x_{1} x_{2}$ : (a) $c=0.1$ and (b) $c=0.5$. 

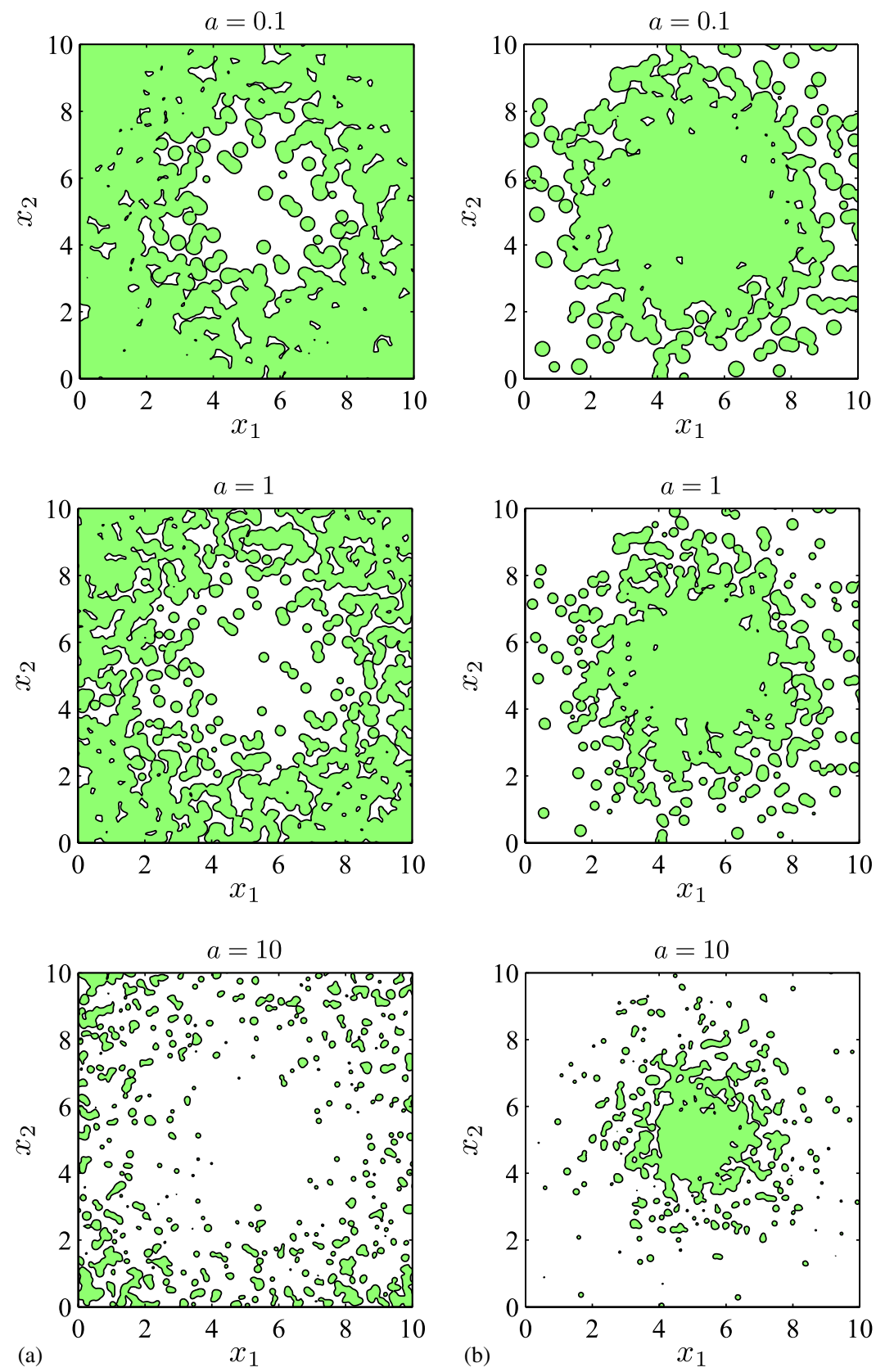

Figure 4. Samples of two-dimensional microstructures of functionally graded composites: (a) centrifugal variation with $c=1$ and (b) anticentrifugal variation with $c=300$. 
anticentrifugal variation. In both variations, three levels $a=0.1,1$, and 10 have been employed to create microstructures exhibiting several distinct features of the embedded particles. Again, these microstructural features can be calibrated if statistical properties of $Y$ are available.

\subsection{Example 2}

Consider a three-dimensional $(d=3)$ specimen with the bounded domain $\mathscr{D}:=(0,10) \times(0,10) \times$ $(0,10) \in \mathbb{R}^{3}$, where fully penetrable spherical or disk-shaped particles entailing random sizes and/or orientations are embedded in the matrix of an FGM. A diagonally varying intensity function

$$
\lambda(\mathbf{x})=c x_{1} x_{2} x_{3}
$$

characterizes the inhomogeneous Poisson field $\mathscr{N}\left(\mathscr{D}^{\prime}\right)$, where $\mathscr{D}^{\prime}=(0,10.5) \times(0,10.5) \times(0,10.5)$, $\mathbf{x}=\left\{x_{1}, x_{2}, x_{3}\right\}^{\mathrm{T}} \in \mathscr{D}^{\prime} \subset \mathbb{R}^{3}$, and $c$ is the intensity parameter. Two values of $c, 0.0005$ and 0.005 , were selected to represent low and high intensities, respectively. For both magnitudes of intensities,

$$
h(\xi)=\exp \left(-\frac{\xi_{1}^{2}}{\sigma_{1}^{2}}-\frac{\xi_{2}^{2}}{\sigma_{2}^{2}}-\frac{\xi_{3}^{2}}{\sigma_{3}^{2}}\right)
$$

where $\xi=\left\{\xi_{1}, \xi_{2}, \xi_{3}\right\}^{\mathrm{T}} \in \mathbb{R}^{3}, \sigma_{1}=\sigma_{2}=\sigma_{3}=0.3$ for the spherical particles, and $\sigma_{1}=\sigma_{2}=0.3, \sigma_{3}=$ 0.06 for the disk-shaped particles. The random variable $Z_{i}=10\left|U_{i}\right|$, where $U_{i}$ is a standard Gaussian random variable with zero mean and unit variance. The rotation matrix $\mathbf{R}_{i}$ is defined by Equation (26), where the Euler angles $\Phi_{i}, \Theta_{i}$, and $\Psi_{i}$ are uniformly distributed in $[0,2 \pi],[0, \pi]$, and $[0,2 \pi]$, respectively.

Figures 5(a)-(d) exhibit four samples of two-phase, functionally graded composites in $\mathbb{R}^{3}$ obtained by the level-cut filtered Poisson field $Y(\mathbf{x})$ in Equation (17) with $Z(\mathbf{x})$ in Equation (2) for level $a=0.01$. The microstructures in Figures 5(a) and (b), which include spherical $\left(\sigma_{1}=\sigma_{2}=\right.$ $\left.\sigma_{3}=0.3\right)$ particles, correspond to low $(c=0.0005)$ and high $(c=0.005)$ values, respectively, of the Poisson intensity. The higher the value of the Poisson intensity, the higher the particle density, resulting in frequent intersections among particles. The samples in Figures 5(c) and (d) contain disk-shaped $\left(\sigma_{1}=\sigma_{2}=0.3, \sigma_{3}=0.06\right)$ particles, also generated using the same two intensities. The significant difference between particle properties in Figures 5(a)-(d) demonstrates once again the capability of the proposed model in generating a broad range of two-phase inhomogeneous microstructures.

\subsection{Example 3}

The objective of the final example is to illustrate the usefulness of the proposed random field model in determining the statistical characteristics of FGM effective properties. Consider a twodimensional, $10 \mathrm{~cm} \times 10 \mathrm{~cm}$, FGM specimen that contains fully penetrable, circular, silicon carbide $(\mathrm{SiC})$ particles with random sizes and number in an aluminum $(\mathrm{Al})$ matrix. The material phases $\mathrm{SiC}$ and $\mathrm{Al}$ are both linear elastic, and their deterministic elastic properties are listed in Table I. The particles follow a horizontally varying Poisson intensity function

$$
\lambda(\mathbf{x})=c \ln \frac{10.5(1+\varepsilon)}{10.5(1+\varepsilon)-x_{1}}
$$



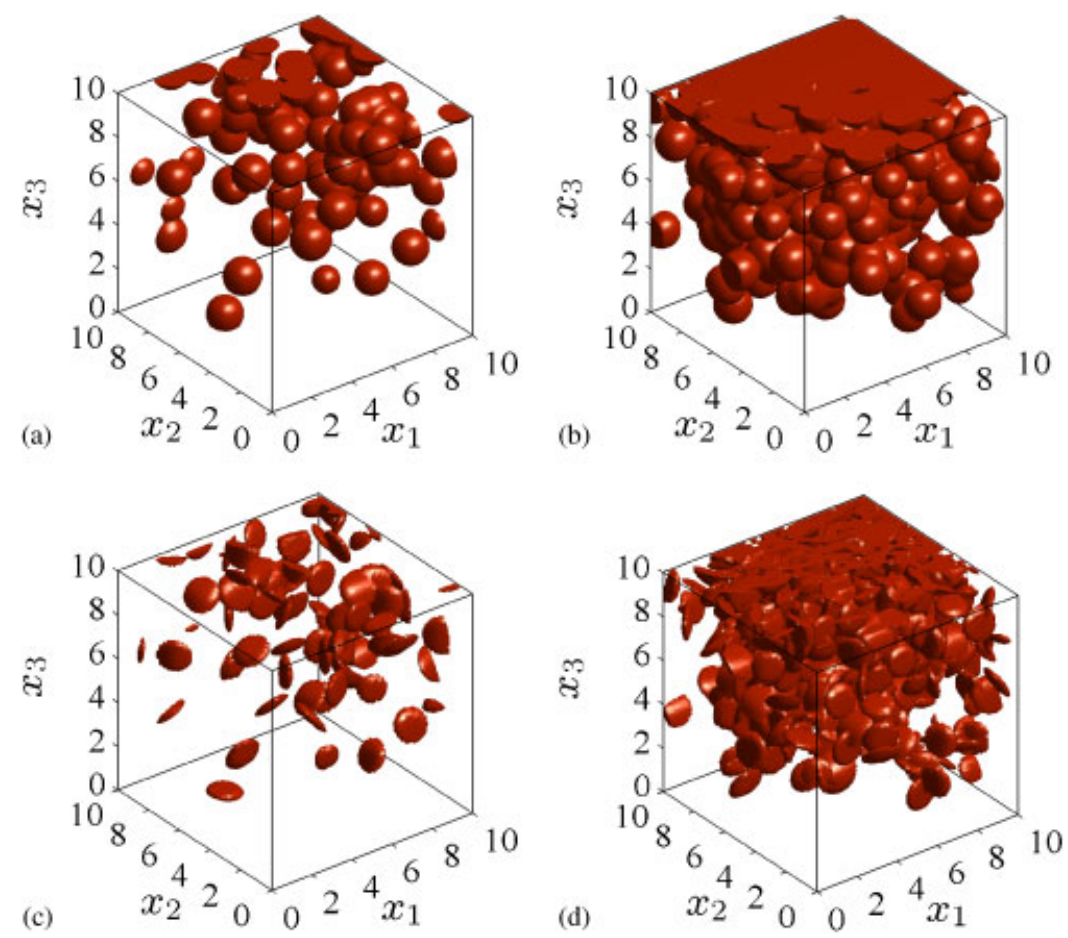

Figure 5. Samples of three-dimensional microstructures of functionally graded composites with a diagonally varying intensity function $\lambda(\mathbf{x})=c x_{1} x_{2} x_{3}$ and $a=0.01$ : (a) spherical particles with $c=0.0005$; (b) spherical particles with $c=0.005$; (c) disk-shaped particles with $c=0.0005 ;$ and (d) disk-shaped particles with $c=0.005$.

Table I. Elastic properties of constituents of SiC-Al FGM.

\begin{tabular}{lcc}
\hline Elastic properties & Silicon carbide $(\mathrm{SiC})$ & Aluminum $(\mathrm{Al})$ \\
\hline Young's modulus, GPa & 419.2 & 69.7 \\
The Poisson's ratio & 0.19 & 0.34 \\
\hline
\end{tabular}

where $\mathbf{x}=\left\{x_{1}, x_{2}\right\}^{\mathrm{T}} \in \mathbb{R}^{2}, 0 \leqslant x_{i} \leqslant 10.5 \mathrm{~cm}, i=1,2, \varepsilon=10^{-6}$, and $c$ is the intensity parameter. The kernel function is given by Equation (28), where $\sigma_{1}=\sigma_{2}=0.2 \mathrm{~cm}$. The random variable $Z_{i}=10\left|U_{i}\right|$, where $U_{i}$ is a standard Gaussian random variable with zero mean and unit variance. The rotation matrix $\mathbf{R}_{i}$ is defined by Equation (25), where the rotation $\Theta_{i}$ is uniformly distributed in [0,2 $\pi$. For the level-cut field, $a=1$.

Figure 6(a) shows a sample of the FGM microstructure employing the level-cut field $Y(\mathbf{x})$ in Equation (17) with $Z(\mathbf{x})$ in Equation (2) and $c=3 \mathrm{~cm}^{-2}$. For comparison, the corresponding sample generated using the approximate random field $\tilde{Y}(\mathbf{x})$ in Equation (23) is presented in Figure 6(b). The microstructural features of both samples in Figures 6(a) and (b) that are relevant to stress analysis are remarkably similar. Such similarity is observed, although to a lesser degree, even when the intensity parameter is raised to a much larger value: $c=30 \mathrm{~cm}^{-2}$, the samples of which are exhibited in Figures $6(\mathrm{c})$ and (d). Note that $\tilde{Y}(\mathbf{x})$ provides a simpler microstructural description 

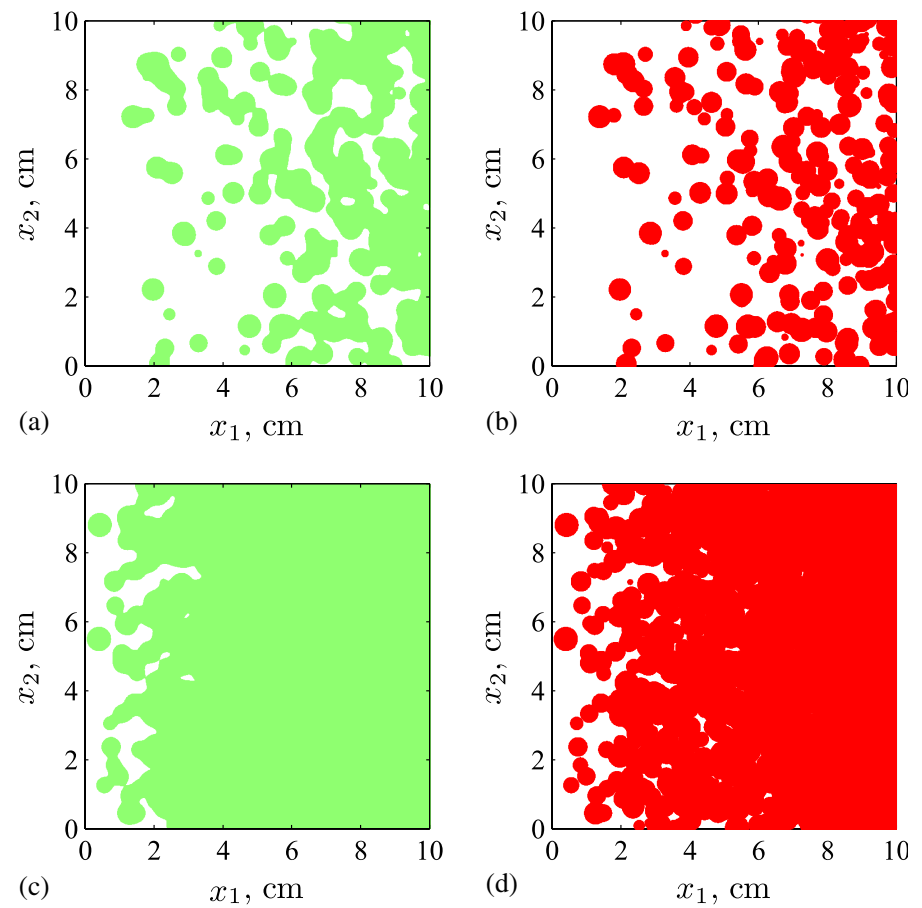

Figure 6. A functionally graded composite with a horizontally varying intensity function: (a) sample of $Y(\mathbf{x})$ with $c=3 \mathrm{~cm}^{-2}$; (b) sample of $\tilde{Y}(\mathbf{x})$ with $c=3 \mathrm{~cm}^{-2}$; (c) sample of $Y(\mathbf{x})$ with $c=30 \mathrm{~cm}^{-2}$; and (d) sample of $\tilde{Y}(\mathbf{x})$ with $c=30 \mathrm{~cm}^{-2}$.

(Equation (24)) that entails defining random particle geometry at each Poisson point. Therefore, a high-quality finite element mesh required for stress analysis can be easily generated using the approximate random field $\tilde{Y}(\mathbf{x})$.

Using $c=3 / \mathrm{cm}^{2}$ and the approximate field $\tilde{Y}(\mathbf{x}), 25$ samples (sample size) of the FGM microstructure were generated. For each microstructural sample, a conforming finite element mesh was created automatically and, therefore, with little effort. Figure 7 shows domain discretizations for four randomly selected samples comprising six-noded, quadratic finite elements. The boundary conditions, sketched in one mesh of Figure 7, include enforcing the simply supported condition at the bottom edge and applying a vertical stress of $1 \mathrm{kN} / \mathrm{cm}^{2}$ to the top edge of the FGM specimen. Subsequently, linear-elastic finite element analyses were performed for all 25 samples, employing the commercial code ABAQUS [21] and a plane stress condition. Figure 8 presents contour plots of the von Mises stress for the aforementioned four samples. The contour plots demonstrate local stress concentrations due to microstructural heterogeneity that vary spatially against the coordinate $x_{1}$, the direction of gradation. However, the stresses vary relatively smoothly on the left side $\left(x_{1}=0\right)$ of the FGM specimen, where there is a dominant presence of the matrix (Al) phase. Perhaps a more useful result is the effective mechanical property, the calculation of which involves two steps. First, the local stresses and strains in each sample were homogenized to calculate the effective stresses and strains on 10 non-overlapping $1 \mathrm{~cm} \times 10 \mathrm{~cm}$ strips that were uniformly deployed in the $x_{1}$ direction. Second, Hooke's law relating effective stresses and strains in each of 

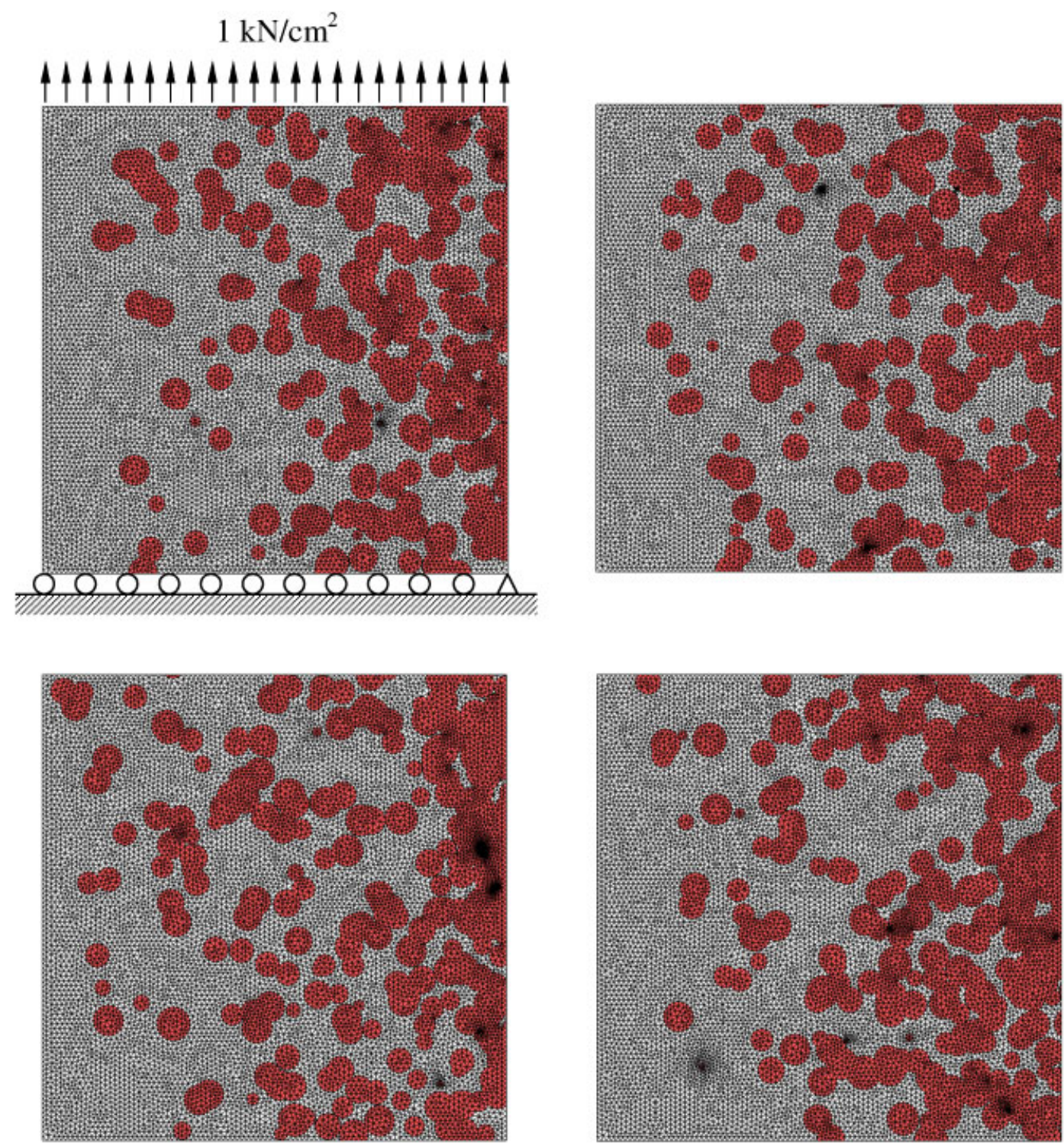

Figure 7. Finite element discretizations for four samples of a SiC-Al FGM.

these 10 strips was invoked to determine the effective elastic modulus and the effective Poisson's ratio. As the intensity function depends only on $x_{1}$ and particles are circular, a resultant elastic property of the composite should vary horizontally, but remain approximately isotropic. ${ }^{\mathbb{I}}$ From

\footnotetext{
"Strictly speaking, a representative volume element should exist to perform such homogenization. Otherwise, a scaledependent homogenization may be required. They were not considered in this paper, because of their complexity. The scale-dependent homogenization was not considered in this example.
} 

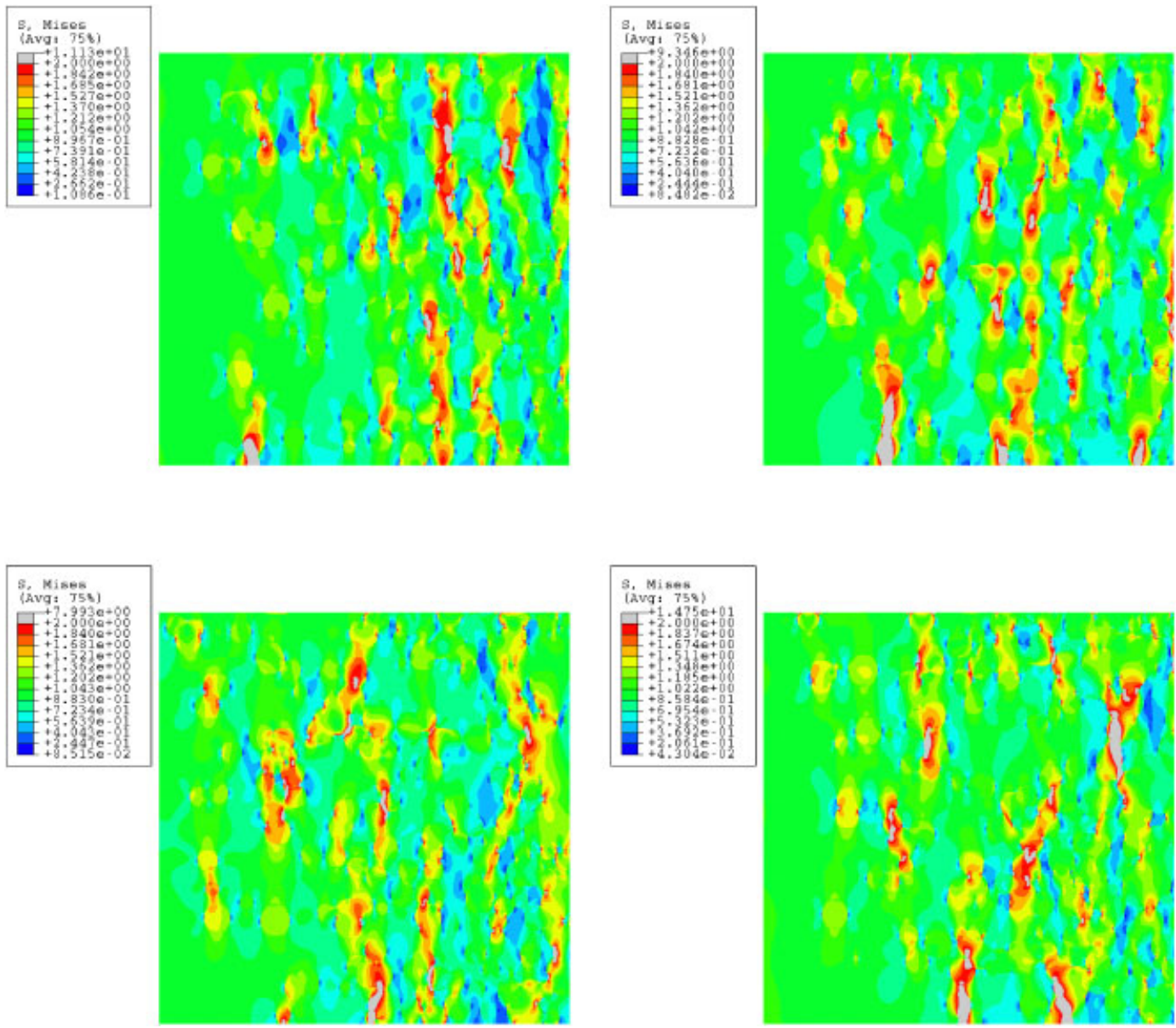

Figure 8. Contour plots of the von Mises stress for four samples of a SiC-Al FGM (unit: $\mathrm{kN} / \mathrm{cm}^{2}$ ).

the Monte Carlo simulation with 25 samples, Figures 9(a) and (b) plot the means and standard deviations of the effective elastic modulus and the effective Poisson's ratio, respectively, of the FGM as functions of $x_{1}$. Such statistics provide useful information on the spatial variation of FGM effective properties, given a random microstructure. Higher-order statistics, including correlation functions, and probability distributions of effective properties can also be estimated by increasing the sample size. Therefore, from the stochastic microstructure-property relationship, if it can be developed, one should be able to design a microstructure to produce target effective properties of FGMs.

\section{LIMITATIONS AND FUTURE WORK}

The random field model described by a single intensity function cannot possibly represent all statistically inhomogeneous microstructures. However, the model can be readily expanded to mimic more 

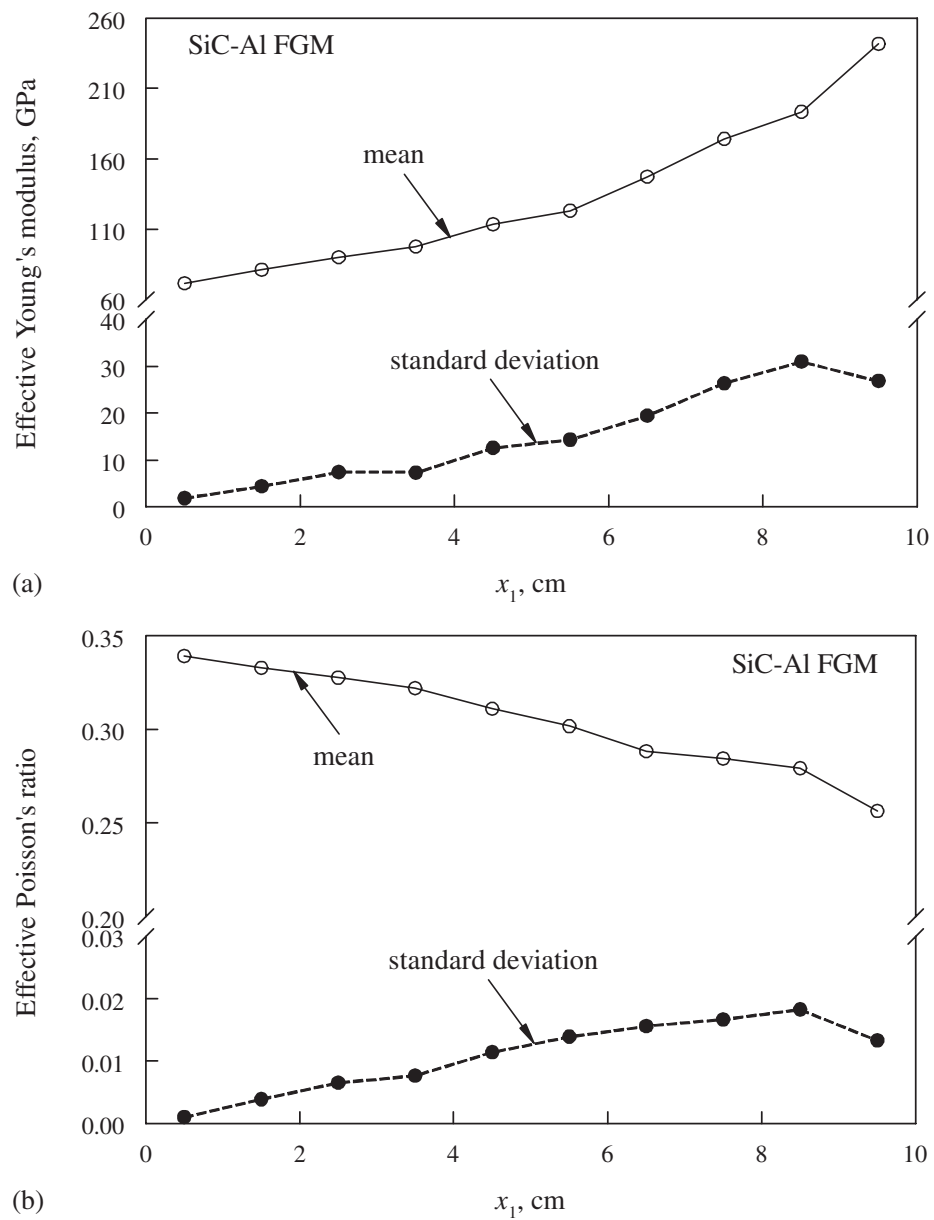

Figure 9. Statistics of effective elastic properties of a SiC-Al FGM: (a) elastic modulus and (b) the Poisson's ratio.

complex morphologies, including percolative thresholds and cellular or non-particulate features. For instance, a two-level cut of $Z(\mathbf{x})$, employing a generalized indicator function $I\left(z ; a_{1}, a_{2}\right):=1$ when $a_{1} \leqslant z \leqslant a_{2}$ and zero otherwise, will permit a constituent phase remains connected down to low volume fractions, commonly exhibited in porous rocks, polymer blends, and polystyrene foams. In addition, from two independent, two level-cut Poisson random fields, the intersection of the volumes of constituent phases will lead to open-cell microstructures of organic aerogels. In contrast, the union of the volumes of constituent phases will produce closed-cell microstructures, encountered in many foamed solids. In other words, a broad range of complex microstructures can be derived from the proposed random field, and their developments are left as future efforts.

In addition, the current paper focuses on modeling the microstructure of the material, where a new stochastic model is proposed. The next challenging problem is to predict the effective properties, such as transport, thermal, mechanical properties, including fracture performance, from 
this microstructural information. Scale-dependent homogenization without relying on representative volume element for predicting effective properties and multiscale fracture simulation properly accounting for microstructural details should be pursued in the future.

\section{CONCLUSIONS}

A level-cut, inhomogeneous, filtered Poisson random field model was developed to represent twophase microstructures of FGMs with fully penetrable embedded particles. The model constitutes a sum of deterministic kernel functions that are scaled by random variables and centered at random points generated by an inhomogeneous Poisson field. The level-cut random fields, defined by the cuts of the inhomogeneous, filtered Poisson random field above the specified levels, can be employed to model random microstructures of statistically inhomogeneous media. The geometric features of the microstructures generated by the model developed depend on the Poisson intensity, level, kernel functions, random scaling variables, and random rotation matrices. The level-cut Poisson field model developed provides an attractive alternative to the commonly employed levelcut Gaussian field model, in which the problem related to the existence of the Gaussian field is avoided. Furthermore, the level-cut Poisson field can be viewed as a generalized random field, which degenerates to the level-cut Gaussian field when the Poisson intensity approaches infinity.

A reconstruction algorithm describing how to calibrate model parameters with available statistical properties of actual microstructural features was developed. The calibration process facilitates optimal evaluations of the model parameters by matching observed correlation functions of an arbitrary order of the level-cut field. Once the model parameters have been determined, a Monte Carlo simulation can be performed to generate samples of microstructures of statistically inhomogeneous media. However, the lack of uniqueness in reconstruction remains a formidable problem, because the use of low-order statistics will always create a class of competing models with two or more members.

Three numerical examples have been presented to illustrate the capability and the usefulness of the level-cut Poisson field model. The results from the first two examples demonstrate that the model developed can create a broad range of two- and three-dimensional microstructures of functionally graded composites containing particles of various sizes, shapes, densities, gradations, and orientations. The third example, involving finite element analyses of random microstructures, provides a means to evaluate statistical characteristics of effective mechanical properties of functionally graded composites. Therefore, microstructures of graded composites can be tailored to meet the desired mechanical properties.

\section{ACKNOWLEDGEMENTS}

The author would like to acknowledge the financial support from the U.S. National Science Foundation under Grant No. CMS-0409463. The author also thanks Mr A. Chakraborty for performing the finite element analysis in Example 3.

\section{REFERENCES}

1. Aurenhammer F. Voronoi diagrams - a survey of a fundamental geometric data structure. ACM Computing Surveys 1991; 23(3):345-405. 
2. Torquato S. Random Heterogeneous Materials: Microstructures and Macroscopic Properties. Springer: New York, NY, 2002.

3. Lu BL, Torquato S. $n$-Point probability functions for a lattice model of heterogeneous media. Physical Review B 1990; 40:4453-4459.

4. Blumenfeld R, Torquato S. Coarse graining procedure to generate and analyze heterogeneous materials - theory. Physical Review E 1993; 48:4492-4500.

5. Roberts AP. Statistical reconstruction of three-dimensional porous media from two-dimensional images. Physical Review E 1997; 56(3):3203-3212.

6. Roberts AP, Teubner M. Transport in heterogeneous materials derived from Gaussian random fields: bounds and simulation. Physical Review E 1995; 51:4141-4154.

7. Berk NF. Scattering properties of the leveled-wave model of random morphologies. Physical Review A 1991; 44:5069-5079.

8. Grigoriu M. Random field models for two-phase microstructures. Journal of Applied Mechanics 2003; 94(6): 3762-3770.

9. Yeong CLY, Torquato S. Reconstructing random media. Physical Review E 1998; 57(1):495-506.

10. Yeong CLY, Torquato S. Reconstructing random media. II. Three-dimensional media from two-dimensional cuts. Physical Review E 1998; 58(1):224-233.

11. Koutsourelakis PS, Deodatis G. Simulation of binary random processes with applications to two-phase random media. Journal of Engineering Mechanics 2005; 131(4):397-412.

12. Grigoriu M. Applied Non-Gaussian Processes: Examples, Theory, Simulation, Linear Random Vibrations, and MATLAB Solutions. Prentice-Hall: Englewood Cliffs, NJ, 1995.

13. Grigoriu M, Garboczi E, Kafali C. Spherical harmonic-based random fields for aggregates used in concrete. Powder Technology 2006; 166:123-138.

14. Suresh S, Mortensen A. Fundamentals of Functionally Graded Materials. IOM Communications Ltd.: London, U.K., 1998.

15. Markworth AJ, Ramesh KS, Parks Jr WP. Modelling studies applied to functionally graded materials. Journal of Materials Science 1995; 30(9):2183-2193.

16. Aboudi J, Pindera M-J, Arnold SM. Higher-order theory for functionally graded materials. Composites, Part B: Engineering 1999; 30:777-832.

17. Rahman S, Chakraborty A. A stochastic micromechanical model for elastic properties of functionally graded materials. Mechanics of Materials 2007; 39:548-563.

18. Gelhar LW. Stochastic Subsurface Hydrology. Prentice-Hall: Englewood Cliffs, NJ, 1993.

19. Bond JR, Kofman L, Pogosyan D. How filaments of galaxies are woven into the cosmic web? Nature 1996; 380:603-606.

20. Quintanilla J, Torquato S. Microstructure functions for a model of statistically inhomogeneous random media. Physical Review E 1997; 55(2):1558-1565.

21. ABAQUS. User's Guide and Theoretical Manual, Version 6.6. ABAQUS, Inc.: Providence, RI, 2006. 